\title{
Evolution of icosahedral copper particles in the process of their growth during electrocrystallization
}

\author{
A. A. Vikarchuk, N. N. Gryzunova ${ }^{\dagger}$ T. A. Borgardt \\ †'gryzunova-natalja@yandex.ru
}

Togliatti State University, 14 Belorusskaya St., Togliatti, 445020, Russia

\begin{abstract}
Metal icosahedral microparticles with six fifth-order symmetry axes, a specific faceting and high catalytic activity, open up wide opportunities for their usage as catalysts capable of operating in fluidized bed reactors. In this paper, microparticles of copper of the icosahedral habitus about 15 microns in size in the form of truncated icosahedra have been grown for the first time by electrodeposition from sulfuric acid phate electrolyte. Such icosahedra are faceted by twelve $\{110\}$ crystallographic planes and twenty $\{111\}$ planes. Studies of the morphology of the surface of copper truncated icosahedra using metallography and electron microscopy have shown that in the process of growth they transform into perfect icosahedra faceted only by $\{111\}$ planes. The structure of icosahedral copper particles has been studied in detail. Electron microscopic studies of the cross section of copper icosahedral particles show that they consist of sub-structural volume elements (fragments) that are separated from each other by twin and (or) dislocation boundaries. Often inside the fragments there are broken boundaries, near which extinction contours of dislocation origin are observed. In addition, coherent twin boundaries of the $\{111\}<112>$ type are observed inside the fragments; they do not create long-range stress fields, twin layers, insertions and dislocations. Pentagonal pits appear on the surface of particles in the process of electrodeposition or etching, which indicate the presence of partial disclinations there. According to the results of experimental data, a scheme for the evolution of truncated icosahedra in the process of copper electrocrystallization into perfect icosahedra is proposed. Using the disclination approach, the density of the total free energy of a truncated and perfect icosahedron has been estimated and it is shown that the transformation of a truncated icosahedron into a perfect one is energetically justified.
\end{abstract}

Keywords: electrodeposition of copper, icosahedral microparticles, pentagonal symmetry.

УДК: $544-971.2,53.096$

\section{Эволюция икосаэдрических частиц меди в процессе их роста при электрокристаллизации}

\author{
Викарчук А. А., Грызунова Н.Н. ${ }^{\dagger}$, Боргардт Т. А. \\ †gryzunova-natalja@yandex.ru
}

Тольяттинский государственный университет, ул. Белорусская, 14, Тольятти, 445020, Россия

Металлические икосаэдрические микрочастицы, имеющие шесть осей симметрии пятого порядка, специфическую огранку и высокую каталитическую активность, предоставляют широкие возможности применения в качестве катализаторов, способных работать в реакторах по технологии «псевдоожиженного слоя». В данной работе электролитическим методом из сернокислого электролита впервые были выращены микрочастицы меди икосаэдрического габитуса размером около 15 мкм в виде усеченных икосаэдров. Такие икосаэдры огранены двенадцатью кристаллографическими плоскостями типа $\{110\}$ и двадцатью плоскостями $\{111\}$. Исследования морфологии поверхности медных усеченных икосаэдров с использованием металлографии и электронной микроскопии показали, что они в процессе роста трансформируются в совершенные икосаэдры, ограненные только плоскостями типа $\{111\}$. В работе детально исследовано строение икосаэдрических частиц меди. Электронно-микроскопические исследования сечения частиц показали, что они состоят из субструктурных объемных элементов (фрагментов), которые отделены друг от друга двойниковыми и (или) дислокационными границами. Часто внутри фрагментов наблюдаются оборванные границы, вблизи которых видны экстинционные контура, имеющие дислокационное происхождение. Кроме того, в частицах наблюдаются когерентные двойниковые границы типа $\{111\}<112>$, не создающие дальнодействующих полей напряжений, двойниковые прослойки, вставки и дислокации. На поверхности частиц в процессе 
электроосаждения или травления появляются пентагональные ямки, что свидетельствует о присутствии там частичных дисклинаций. По результатам экспериментальных данных предложена схема эволюции в процессе электрокристаллизации меди усеченных икосаэдров в совершенные икосаэдры. Используя дисклинационный подход, была произведена оценка плотности полной свободной энергии усеченного и совершенного икосаэдра и показано, что превращение усеченного икосаэдра в совершенный энергетически оправдано.

Ключевые слова: электроосаждение меди, икосаэдрические микрочастицы, пентагональная симметрия.

\section{1. Введение}

Металлические икосаэдрические частицы (ИЧ), имеющие шесть осей симметрии пятого порядка, вызывают повышенный интерес исследователей, как теоретиков, так и экспериментаторов, во всем научном мире [1-6]. Специфическая огранка, дефектная структура и необычные свойства открывают широкие возможности их применения в различных отраслях промышленности, в частности, их можно использовать в качестве катализаторов, способных работать в реакторах по технологии «псевдоожиженного слоя» $[7,8]$.

Теория [1,3,5,9-11] предсказывает, что при наноразмерах в металлических частицах энергетически выгодно икосаэдрическое расположение атомов, но при достижении некоторого критического размера (порядка 50 - 60 нм) наночастица должна превратиться в кристалл. Однако на практике как авторы настоящей работы, так и другие исследователи [10,13-15] наблюдали ИЧ, вырастающие и сохраняющие форму до десятков микрометров (Рис. 1). Как правило, такие крупные металлические ИЧ получались методом электроосаждения [10,13-15].

По мнению авторов [10,13-15], образование и рост наночастиц с икосаэдрическим расположением атомов в процессе электроосаждения металлов происходит из трехмерных кластеров (Рис. S1a (дополнительный материал)). Далее из некристаллических наночастиц (Рис. S1b) в зависимости от условий электролиза, состава электролита, природы подложки могут вырастать микрокристаллы с икосаэдрическим габитусом (для меди размером порядка 20 - 30 мкм) в виде звездчатых пентагональных многогранников, совершенных икосаэдров, а также икосаэдрических микрочастиц неправильной формы (Рис. S1c). В настоящей работе впервые экспериментально обнаружено и исследовано превращение в процессе роста при электрокристаллизации меди сферических наночастиц в усеченные икосаэдры, а последних в совершенные икосаэдры микронных размеров.

\section{2. Методика получения и исследования}

Для получения ИЧ меди различной формы использовался сернокислый электролит меднения без добавок, приготовленный на бидистиллате из химически чистых компонентов и содержащий 250 г/л, $\mathrm{CuSO}_{4} \cdot 5 \mathrm{H}_{2} \mathrm{O}$ и 90 г/л $\mathrm{H}_{2} \mathrm{SO}_{4}$. Электроосаждение осуществлялось в трехэлектродной ячейке с помощью гальваностата "Elins" в потенциостатическом режиме, и использовались следующие параметры осаждения: перенапряжение $-85 \pm 5 \mathrm{mV}$, время осаждения - от 20 сек. до 20 мин., $\mathrm{pH}$ электролита - 1.3-1.4, температура $20-25^{\circ} \mathrm{C}$. Анод был изготовлен из электролитической чистой меди (99.99\%), а в качестве катода и одновременно подложки применялась полированная нержавеющая сталь марки 12Х18Н10 или микросетка из нержавеющей стали той же марки. Подложка предварительно обрабатывалась в изопропиловом спирте в ультразвуковой ванне, затем промывалась в дистиллированной воде и высушивалась.

Исследование морфологии поверхности проводили с использованием сканирующей электронной микроскопии (Zeiss Sigma) и металлографии (Zeiss Axiovert 40 MAT). Структуру изучали, используя просвечивающий микроскоп Tесnai Osiris в Центре коллективного пользования Института проблем химической физики Российской академии наук (ИПХФ РАН).

\section{3. Результаты и их обсуждение}

Электронно-микроскопические исследования показали, что при выбранных условиях электроосаждения, через 20 - 30 секунд на поверхности подложки из нержавеющей стали формируются сферические некристаллические наночастицы меди (Рис. S1b (дополнительный материал), Рис. 1a), из которых через 15 минут вырастают микрочастицы икосаэдрического габитуса (ИЧ) (Рис. S1c, дополнительный материал). Большинство ИЧ, полученных при указанных режимах электроосаждения, имеют в размерном диапазоне от 5 до 15 мкм форму архимедовых усеченных икосаэдров (УИ) (Рис. 1b, с) [16]. Замечено, что в процессе дальнейшего роста УИ превращаются в совершенные икосаэдры (СИ) (Рис. 1d). В данном случае при росте ИЧ меди реализуется следующая последовательность: сферические наночастицы с икосаэдрическим расположением атомов - усеченные икосаэдры, ограненные 32 атомными плоскостями типа $\{110\}$ и $\{111\}$ - совершенные икосаэдры, ограненные только плоскостями $\{111\}$ (Рис. 1). Такая эволюция при росте ИЧ меди была замечена впервые.

Детальные исследования роста медных УИ показали, что они вырастают из сферических наночастиц, не имеющих огранки (Рис. 1a, Рис. S1b, дополнительный материал). В процессе дальнейшего роста такие частицы приобретают 6 осей симметрии пятого порядка, 60 вершин, 32 грани и 90 ребер, т. е. из наночастиц образуются УИ микронного размера (Рис. 1a, b, c).

Сначала на поверхности УИ наблюдаются 12 пятии 20 шести- правильных многоугольников (Рис. 1b). Пятиугольные грани имеют вид вицинальных поверхностей (Рис. 1c), состоящих из террас и ступеней роста, а шестиугольники имеют несингулярную поверхность [17]. Оба вида граней обладают по сравнению с другими атомными плоскостями повышенной поверхностной энергией [17], что противоречит закону роста кристаллов, согласно которому поверхностная энергия должна 


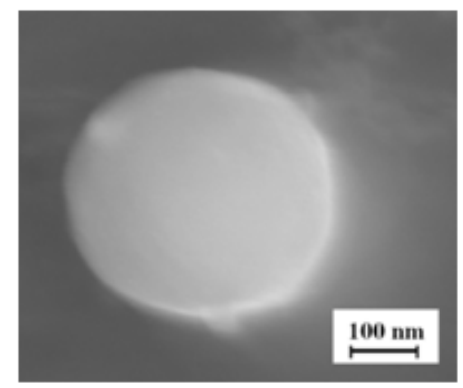

a

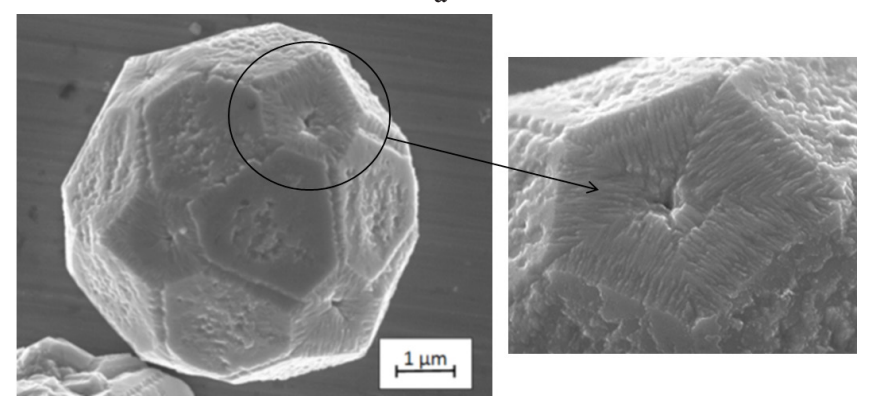

C

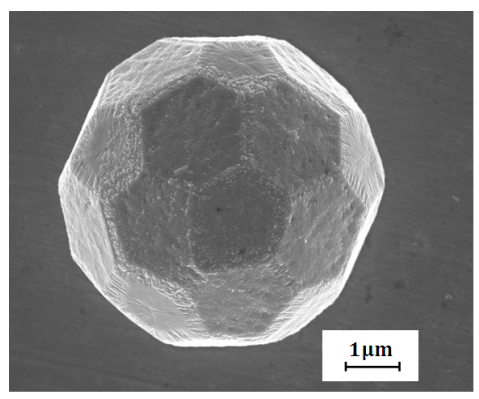

b
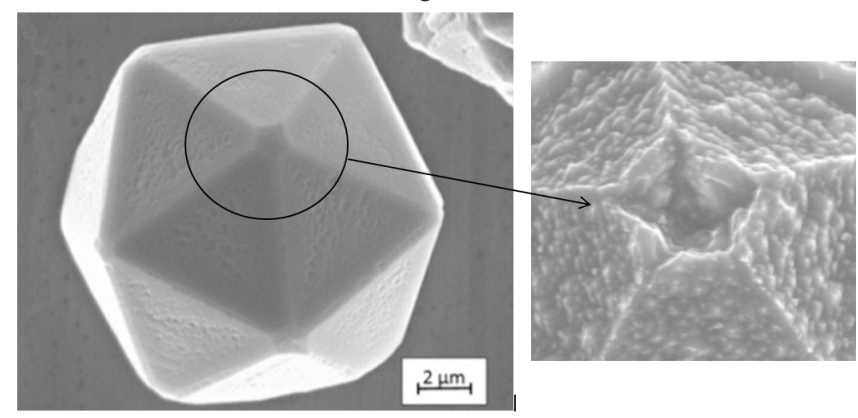

d

Рис. 1. Эволюция усеченных икосаэдров в совершенные икосаэдры в процессе электрокристаллизации меди (а)-(d) и ямки, образовавшиеся на поверхности УИ и СИ при электроосаждении (c) и химическом травлении (d).

Fig. 1. The evolution of truncated icosahedra in perfect icosahedra during electrocrystallization of copper (a)-(d) and pits formed on the surface of the TI and PI during electrodeposition (c) and chemical etching (d).

быть минимальной [18]. В вершинах УИ сходятся два шестиугольника и один пятиугольник, каждый из пятиугольников окружён шестиугольниками (Рис. 1b,c). Кристаллографический анализ методом дифракции обратно-рассеянных электронов от граней УИ показал, что пятиугольники являются кристаллографическими гранями типа $\{110\}$, а шестиугольники и треугольники на поверхности частиц - это атомные плоскости $\{111\}$ (Рис. S2 a, дополнительный материал).

Эксперименты показали (Рис. 1), что в процессе формирования УИ при электрокристаллизации преимущественный рост имеют пятиугольные высокоэнергетические грани, состоящие из атомных плоскостей $\{110\}$, на которые, по нашему мнению, выходят частичные дисклинации (Рис. 1с). Выход частичных дисклинаций на плоскости $\{110\}$ проявляется в виде ямок, формирующихся при электроосаждении (Рис. 1с).

В процессе роста пятиугольники на поверхности УИ зарастают, а 20 шестиугольников, состоящих из кристаллографических плоскостей $\{111\}$, трансформируются в треугольники (Рис. 1c, d, Рис. S3 a, дополнительный материал). Зарастание пятиугольных граней УИ типа $\{110\}$ и превращение 20-ти шестиугольных граней типа $\{111\}$ в треугольные (Рис. 1c) сопровождается миграцией 60-ти границ между фрагментами, опирающимися на ребра пятиугольников и шестиугольников (Рис. 1b, c, Рис. S4). Электронно-микроскопические (Рис. 2, Рис. S3, S4) и металлографические (Рис. S4) исследования икосаэдрических частиц меди подтвердили, что они состоят из объемных структурных элементов: совершенный икосаэдр - из 20 кристаллических тетраэдров, а УИ - из 32 конусообразных объемных элементов, в основании которых лежат правильные пяти- или шестиугольники. Подтверждения данного утверждения приведены на Рис. 2 и Рис. S2, S3, S4. В дополнительном материале (Рис. S3) представлена методика изготовления фольг из икосаэдрических частиц для проведения электронно-микроскопических исследований. Сначала на икосаэдрческую частицу (Рис. 2a, Рис. S3 b) наносился тонкий слой тугоплавкого металла, затем из медной частицы, покрытой оболочкой из тугоплавкого металла, готовилась с помощью ионной пушки фольга (Рис. S3 b), которая утонялась и исследовалась в электронном микроскопе на просвет. Видно (Рис. 2 a, Рис. S3c), что частица состоит из субструктурных объемных элементов конусообразной формы, сходящихся в центре частицы и разделенных между собой большеугловыми границами типа $<112>\cdot\{111\}$. Места обрыва границ раздела - это частичная дисклинация, создающая дальнодействующие поля напряжений (это видно по экстинционным контурам, имеющим дислокационную природу (Рис. 2 a, Рис. S3 c) [24]. Исследования пленок, вырезанных из совершенного икосаэдра, показало, что электронограммы от центра частицы демонстрируют пятерную симметрию (характерную для наночастицы (Рис. S1b), электронограммы от границ раздела соответствуют двойниковым границам (Рис. S2 b), а электронограммы от фрагментов соответствуют ГЦК-решетке (Рис. 2 b, с).

Детальные электронно-микроскопические исследования сечения медных микрочастиц в виде УИ и СИ показали (Рис. 2 a, Рис. S3 c, дополнительный материал), что они содержат большое количество обрывающихся двойниковых границ, вблизи которых наблюдаются экстинционные контура, имеющие дислокационное происхождение (Рис. 2a) и свидетельствующие о наличии там частичных дисклинаций [19]. Имеются также двойниковые границы типа $<112>\{111\}$, не создающие дальнодействующих полей напряжения (Рис. 2 a, b, Рис. S2 b). 


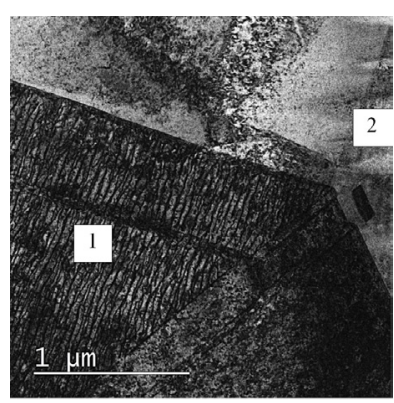

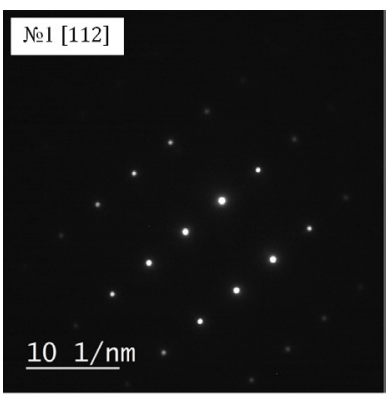

b

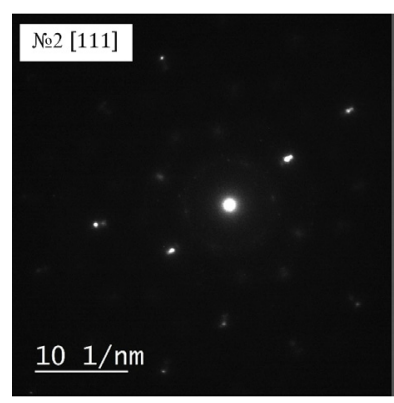

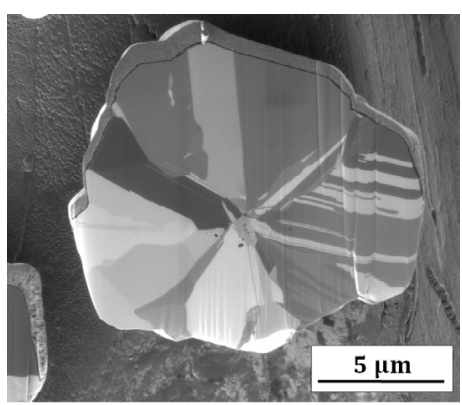

d

Рис. 2. Микрофотография сечения ИЧ меди (а), электронограммы от разориентированных участков 1 и 3 (b), (c), микродифракции получены от области 0,1 мкм, ось зоны 101; микрофотография от среза ИЧ (d).

Fig. 2. A micrograph of the cross section of the copper IP (a), the electron diffraction pattern from the misoriented sections (b), (c), microdiffraction is obtained from the region of $0.1 \mu \mathrm{m}$, the axis of zone 101; photomicrograph from the cut of the IP (d).

ИЧ содержат кроме двойниковых границ двойниковые прослойки и вставки (Рис. $2 \mathrm{a}, \mathrm{d}$, Рис. S3c)

Таким образом, мы можем утверждать, что СИ состоит из объемных структурных элементов (фрагментов). Фрагменты имеют вид тетраэдров. В вершинах СИ находятся частичные дисклинации.

Что касается усеченных икосаэдров, электронно-микроскопические и металлографические исследования показали, что они состоят из 32-х объемных структурных конических элементов, разделенных между собой 30-ю двойниковыми границами (такими же, как и в совершенном икосаэдре) и 60-ю малоугловыми дислокационными границами, залегающими в плоскостях $<112>$. Доказать последнее утверждение, используя электронную микроскопию, не удалось, так как при утонении фольги, вырезанной из УИ, дислокационные границы рассыпались. Однако на металлографических снимках было зафиксировано, что в кристаллических шестиугольниках содержатся двойниковые границы, прослойки, вставки и дислокации (Рис. S4c, дополнительный материал). Пентагональные объемные элементы содержали частичные дисклинации (Рис. 1с), их решетка была крайне искажена.

Замечено, что в процессе роста УИ в центре пятиугольников (на плоскостях типа $\{110\}$ ) появляются и увеличиваются ямки травления ростового происхождения (Рис. 1с), в которых сходятся пять двойниковых границ (Рис. 1с и Рис. 3 d), т.е. там, где выходят на поверхность пятиугольников УИ частичные дисклинации. Наличие дисклинаций и обрывающихся на них двойниковых границ, а также входящих углов, образованных двойниковыми границами, высокая поверхностная энергия вицинальных атомных плоскостей [17] обеспечивают в УИ опережающий рост пятиугольных граней по сравнению шестиугольными (Рис. $1 \mathrm{~b}, \mathrm{c}$ ). Рост частиц осуществляется за счет поступления на их поверхность ионов меди из объема электролита, а также диффузии адатомов по поверхности частицы к границам раздела и выходам дисклинаций в пентагональных конусообразных фрагментах. При этом грани пятиугольной формы, от периферии к центру, начинают интенсивно зарастать (Рис. 1c), а грани $\{111\}$ трансформируются из шестиугольников в треугольники (Рис. 1b,c). Двойниковые границы являются специальными, типа $\Sigma 3$ и стабиль- ными (Рис. 3b), они только удлиняются за счет перемещения неравновесных границ между структурными элементами, опирающимися на пяти- и шестиугольники (Рис. 1c, Рис. 3 с). Эти границы, по-видимому, являются малоугловыми дислокационными, т. к. на электронномикроскопических снимках мы обнаружили высокую плотность дислокаций, залегающих в плоскостях $\{112\}$ (Рис. 2а). В процессе роста УИ дислокационные границы мигрируют (Рис. 1с) по диффузионному механизму [20] за счет поступающих к ним точечным дефектам, образующимся в процессе электрокристаллизации меди (в процессе электрокристаллизации меди образуется порядка $10^{-4}$ неравновесных вакансий [21]). Возможный механизм образования двойниковых и дислокационных границ, а также двойниковых прослоек в нанокристаллах за счет дальнодействующих полей напряжений от дисклинаций, детально описан в работах [22-24]. Таким образом, можно утверждать, что УИ также состоит из структурных объемных элементов (Рис. S4, дополнительный материал). УИ, по металлографическим данным (Рис. S4), состоит из 12 сильно искаженных дисклинацией пентагональных участков, окруженных 20-ю кристаллическими областями (Рис. 1b,c). 12 пентагональных участков необходимы для того, чтобы сохранить в их объеме кристаллическое состояние и обеспечить на поверхности частицы необходимую кривизну [26].

На Рис. 3 предложена схема эволюции в процессе электрокристаллизации меди напряженной сферической наночастицы с икосаэдрическим расположением в ней атомов $[5,25]$ в усеченный икосаэдр, ограненный кристаллографическими плоскостями $\{111\}$ и $\{110\}$, а затем УИ в совершенный икосаэдр, имеющий только грани типа $\{111\}$.

Используя данные для меди, приведенные в работах [22, 25, 27-29], и, приняв, что удельная энергия для малоугловой дислокационной границы раздела между пяти- и шестиугольниками примерно равна $10 \cdot \gamma_{\text {Дв }}$ можно провести оценку объемной плотности энергии растущих частиц (см. Табл. 1 и дополнительный материал). Из Табл. 1 видно, что при одном и том же размере частицы, имеющие форму УИ, обладают более высокой объемной плотностью энергии, чем частицы в виде СИ. Это позволяет говорить о том, что УИ имеет нестабильную морфологическую форму ИЧ, его преобразование в СИ является энергетически выгодным. 


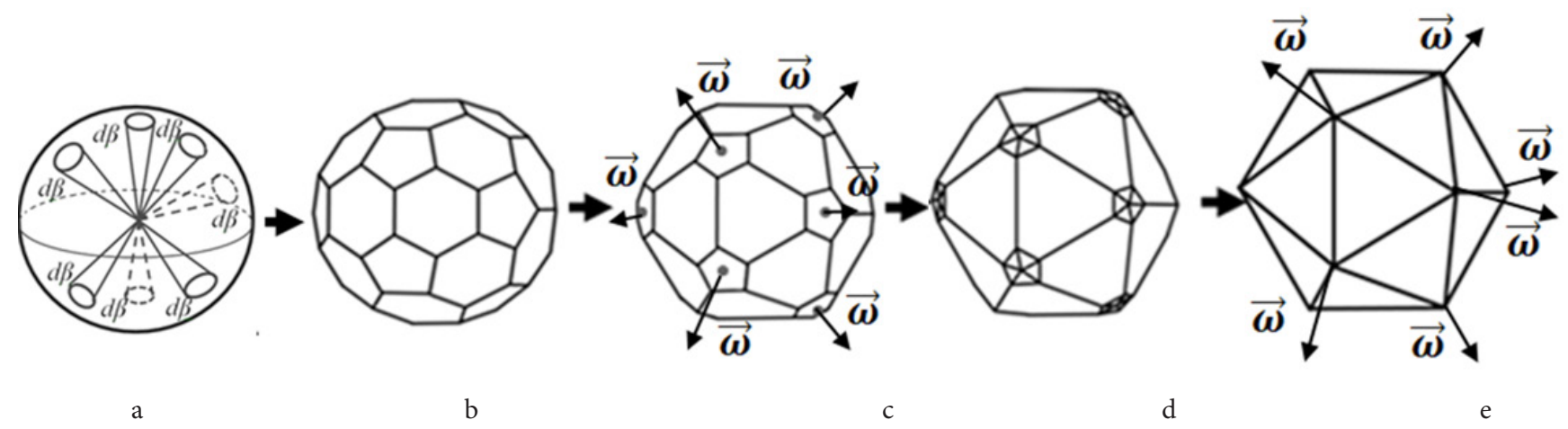

Рис. 3. Дисклинационная схема формирования в процессе электрокристаллизации меди из сферических наночастиц УИ, а из него СИ. Fig. 3. Disclination scheme for the formation of copper in the process of electrocrystallization from spherical nanoparticles of a TI, and from it a PI.

табл. 1. Сравнение плотностей энергий частиц.

Table 1. Comparison of energy density of particles.

\begin{tabular}{|c|c|c|c|}
\hline Радиус $\quad \begin{array}{c}\text { Тип частицы } \\
\text { Type of particle }\end{array}$ & \multicolumn{3}{|c|}{$\begin{array}{l}\text { Плотность энергии частиц } \\
\text { Particle energy density }\end{array}$} \\
\hline Radius of the particle, $\mu \mathrm{m}$ & $\begin{array}{c}\text { Наночастица (НЧ) } \\
\text { Nanoparticle (NP) }\end{array}$ & $\begin{array}{l}\text { Усеченный икосаэдр (УИ) } \\
\text { Truncated icosahedron (ТІ) }\end{array}$ & $\begin{array}{c}\text { Совершенный икосаэдр (СИ) } \\
\text { Perfect icosahedron (PI) }\end{array}$ \\
\hline 0.1 & $G / 325$ & - & - \\
\hline 1.0 & G/430 & $G / 700$ & $G / 720$ \\
\hline
\end{tabular}

Таким образом, анализ экспериментов позволяет сделать выводы:

1. В процессе роста икосаэдрических частиц меди при электрокристаллизации при определенных условиях реализуется следующая последовательность превращения: сферическая наночастица (существует до размеров порядка 0.3-0.5 мкм) - усеченный икосаэдр (до размеров 10-15 мкм) - совершенный икосаэдр (до 20 - 25 мкм)

2. Совершенный икосаэдр состоит из 20 весьма совершенных кристаллических тетраэдрических областей, разделенных между собой двойниковыми границами, он огранен только кристаллографическими плоскостями $\{111\}$, имеет 12 вершин и содержит 6 частичных клиновых дисклинаций мощностью $7^{\circ} 20^{\prime}$.

3. Усеченный икосаэдр состоит из 12 крайне искажённых кристаллических областей в виде пятиугольных призм, содержащих частичную дисклинацию. Каждый пятиугольник окружен кристаллическими областями в виде шестиугольников. Шестиугольники и пятиугольники разделены между собой малоугловыми дислокационными границами. Шесть частичных дисклинаций в УИ ориентированы вдоль осей симметрии, проходящих через пятиугольники.

4. Плотность свободной объемной и поверхностной энергии в наночастице, усеченном и совершенном икосаэдре в процессе их роста и преобразования уменьшается, что говорит об энергетической выгодности такой трансформации. При этом вероятность появления из наночастицы совершенного или усеченного икосаэдра примерно одинакова.

\section{4. Заключение}

Полученные данные позволяют объяснить эволюцию формирования медных икосаэдрических ча- стиц в процессе электрокристаллизации: на подложке из икосаэдрических кластеров образуются наночастицы сферической формы, затем из них формируются при определенных условиях икосаэдрические микрочастицы, в частности УИ, а из последних вырастают СИ.

При наноразмерах энергетически выгодно образовываться наночастицам, имеющих икосаэдрическое расположение атомов. В процессе роста частицы, растет ее упругая энергия, которая релаксируется путем фрагментации, с образованием из сферической некристаллической наночастицы УИ, состоящего из 32 структурных объемных элементов: 12 пятиугольных и 20 шестиугольных призм, УИ содержит 6 частичных дисклинаций, расположенных по осям симметрии пятого порядка. В процессе роста УИ трансформируется в СИ, состоящий из 20 тетраэдров, ограненных кристаллографическими плоскостями $\{111\}$ и содержащего 6 частичных клиновых дисклинаций. Трансформация УИ в СИ хорошо описывается в рамках дисклинационных моделей, энергетически выгодна и экспериментально наблюдается в процессе электрокристаллизации меди.

Благодарности/Acknowledgements. Работа была выполнена в рамках государственного задания Министерства образования и науки Российской Федераüu 16.2314.2017/4.6./The work was carried out within the framework of the state task of the Ministry of Education and Science of the Russian Federation 16.2314.2017/4.6.

Дополнительный материал/Supplementary Material. Электронная версия статьи содержит дополнительный материал, доступный безвозмездно на сайте журнала (lettersonmaterials.com)./The online version of this paper contains supplementary material available free of charge at the journal's Web site (lettersonmaterials.com). 


\section{Литература/References}

1. V.G. Gryaznov, J. Heidenreich, A.M. Kaprelov, S.A. Nepijko, A.E. Romanov, J. Urban. Crystal Research and Technology. 34, 1091 (1999). DOI: $\quad 10.1002 /(S I C I) 1521-4079(199911) 34: 9<1091:: A I D-$ CRAT1091>3.0.CO;2-S

2. X. Yang, K.D. Gilroy, M. Vara, M. Zhao, S. Zhou, Y. Xia. Chemical Physics Letters. 683, 613 (2017). DOI: 10.1016/j.cplett.2017.01.040

3. S. Ogawa, S. Ino. Journal of Crystal Growth. 13/14, 48 (1972).

4. L. D. Howie. Marks Philosophical Magazine A. 1 (49), 95 (1984).

5. L.D. Marks, L. Peng. Journal of Physics: Condensed Matter. 28, 48 (2016).

6. H. Wang, Sh. Zhou, K.D. Gilroy, Z. Cai, Y.Xia. Nano Today. 15, 121 (2017). DOI: 10.1007/s11467-013-0324-x

7. I.V. Mishakov, V.A. Lyholobov. Introduction to catalysis. Novosibirsk, NSU (2015) 67 p. (in Russian) [И. В. Мишаков, В. А. Лихолобов. Введение в катализ. Новосибирск, НГУ (2015) 67 c.]

8. G.V. Meshcheryakov. Proceedings of Tula State University. Natural Sciences. 1 (2), 148 (2014). (in Russian) [Г.В. Мещеряков. Известия ТулГУ. Естественные науки. 1 (2), 148 (2014).]

9. Yu.N. Gornostyrev, I.N. Karkin, M.I. Candzelson, A.V. Trefilov. Physics of metals and metallurgy. 2 (96), 19 (2003). (in Russian) [Ю.Н. Горностырев, И.Н. Карькин, М.И. Канцельсон, А.В. Трефилов. Физика металлов и металловедение. 2 (96), 19 (2003).]

10. A.A. Vikarchuk, I.S. Yasnikov. Structurization in nanoparticles and microcrystals with pentagonal symmetry, formed during electrocrystallization of fcc metals. Togliatti, TSU (2006) 208 p.] (in Russian) [А.А. Викарчук, И.С. Ясников. Структурообразование в наночастицах и микрокристаллах с пентагональной симметрией, формирующихся при электрокристаллизации ГЦКметаллов. Тольятти, ТГУ (2006) 208 с.]

11. A.I. Gusev, A.A. Rempel. Nanocrystalline materials. Moscow, Fizmatlit. (2001) 224 p. (in Russian) [А.И. Гусев, А.А. Ремпель. Нанокристаллические материалы. Москва, Физматлит. (2001) 224 с.]

12. M.Y. Gutkin, A.L. Kolesnikova, I.S. Yasnikov, A.A. Vikarchuk, E.C. Aifantis, A.E. Romanov. European Journal of Mechanics, A/Solids, 68, 133 (2018). DOI: 10.1016/j.euromechsol.2017.11.004

13. I.S. Yasnikov, A.A. Vikarchuk. Metal Science and Heat Treatment. 49 (3-4), 97 (2007). (in Russian) [И.С. Ясников, А.А. Викарчук. Металловедение и термообработка. 49 (3-4), 97 (2007).]

14. I.S. Yasnikov. Letters on Materials. 1, 51 (2011). (in Russian) [И.С. Ясников. Письма о материалах. 1, 51 (2011).] DOI: 10.22226/2410-3535-2011-1-51-54

15. T.A. Ovechkina, N.N. Gryzunova, A.A. Vikarchuk et al. Letters on Materials. 7 (2), 120 (2017). (in Russian) [T.А. Овечкина, Н.Н. Грызунова, А.А. Викарчук и др. Письма о материалах. 7 (2), 120 (2017).] DOI: $10.22226 / 2410-3535-2017-2-120-124$

16. P.S. Aleksandrov, A.I. Markushevich, A.Ya. Khinchin. Polygons and polyhedra. Encyclopedia of elementary mathematics. Fourth edition. Geometriya. Moscow, State Publishing House of Physical and Mathematical Literature. (1963) 382 p. (in Russian) [П.С. Александрова, А.И. Маркушевича, А.Я. Хинчина. Многоугольники и многогранники. Энциклопедия элементарной математики. Книга четвёртая. Геометрия. Москва, Государственное издательство физико-математической литературы. (1963) 382 с.]

17. V.I. Tomilin, N.P. Tomilina, V.A. Bakhtina. Physical Materials Science. Part 1. Passive dielectrics: study guide. Krasnoyarsk, Siberian. federal university. (2012) 280 p. (in Russian) [В.И. Томилин, Н.П. Томилина, В.А. Бахтина. Физическое материаловедение: в 2 ч. Ч. 1. Пассивные диэлектрики: учеб. пособие. Красноярск, Сиб. федер. ун-т. (2012) 280 c.]

18. The process of real crystal formation. Ed. by N.V. Belova. Moscow, Nauka. (1977) 235 p. (in Russian) [Процессы реального кристаллообразования. Под ред. Н.В. Белова. Москва, Наука. (1977) 235 с.]

19. E.V. Kozlov, A.M. Glezer, N.A. Koneva, N.A. Popova, I.A. Kurzina. Fundamentals of plastic deformation of nanostructured materials. Moscow, Fizmatlit. (2016) 304 p. (in Russian) [Э.В. Козлов, А.М. Глезер, Н.А. Конева, Н.А. Попова, И.А. Курзина. Основы пластической деформации наноструктурных материалов. Москва, Физматлит. (2016) 304 с.]

20. M.A. Shtremel. Strength of alloys. Part 1: Defects of the lattice. Moscow, MISIS. (1999) 384 p. (in Russian) [М.А. Штремель. Прочность сплавов. Ч. 1: Дефекты решетки. Москва, МИСИС. (1999) 384 с.]

21. V.V. Povetkin, I.M. Kovensky. Electrolytic coating structure. Moscow, Metallurgy. (1989) 136 p. (in Russian) [Поветкин В.В., Ковенский И.М. Структура электролитических покрытий. Москва. Металлургия. (1989) 136 с.]

22. I. A. Ovidko, N. V. Skiba. Materials Physics and Mechanics. 21, 288 (2014). (in Russian) [И.А. Овидько, Н. В. Скиба. Физика и механика материалов. 21, 288 (2014).]

23. M.Yu. Gutkin, I.A. Ovidko, N.V. Skiba. Solid State Physics. 46 (11), 1975 (2004). (in Russian) [М.Ю. Гуткин, И.А. Овидько, Н.В. Скиба. Физика твердого тела. 46 (11), 1975 (2004).]

24. G.F. Sarafanov, V.N. Perevezentsev. Letters on Materials. 1 (1), 19 (2011). (in Russian) [Г.Ф. Сарафанов, В.Н. Перевезенцев. Письма о материалах. 1 (1), 19 (2011).] DOI: 10.22226/2410-3535-2011-1-19-24

25. L.M. Dorogin, S. Vlassov, A.L. Kolesnikova, I. Kink, R. Lohmus, and A.E. Romanov. Physica Status Solidi B. 247 (2), 288 (2010). DOI: 10.1002/pssb.200945385

26. A.E. Katz. Energy, economy, technology, ecology. 3, 25 (2002). (in Russian) [А.Е. Кац. Энергия, экономика, техника, экология. 3, 25 (2002).]

27. S. Kibey, J.B. Liu, D.D. Johnson, H. Schitoglu. Acta Materialia. 55, $6843 \quad$ (2007). DOI: 10.1016/j.actamat.2007.08.042.

28. J.P. Hirth, J. Lothe. Theory of Dislocations. New York, Willey. (1982) $435 \mathrm{p}$.

29. V.N. Chuvildiev. Bulletin of the Nizhny Novgorod University. N. I. Lobachevsky. 5 (2), 124 (2010). (in Russian) [В.Н. Чувильдиев. Вестник Нижнегородского ун-та им. Н. И. Лобачевского, 5 (2), 124 (2010). 